\title{
Inhaltsverzeichnifs des vier und vierzigsten Bandes, nach den Gegenständen.
}

\author{
I. Reine Mathematik.
}

Nr. der sblhandlung.

1. A n a l y s i s.

2. Zweiler Nachtrag zu der Theorie der analytischen Facultăten (Bd. 35 und 38 ). Von Herrn Dr. Ottinger, Prof. ord. an der Universität zu Freiburg im Breisgau.

11. Schlufs dieser Abhandlung. . . . . . . . . . . . . . .

I. 26

3. Vereinfachung des Beweises von Cauchy, dafs jede Gleichung nten Grades wenigstens einc Wurzel hat. Von Herrn J. Sufsmunn, Assistent im Königlichen Gewerbe-Institut zu Berlin. . . . . . . . . . . . . . . I. 57

8. Aufgabe, nebst Auflösung. Vom Herausgeber. . . . . . . . . . . I. 88

10. Über die Ergānzungssätze zu den allgemeinen Reciprocitătsgesetzen. Von Herrn Dr. E. E. Kummer, Professor an der Universitãt zu Breslau. . . .

14. Auszug eines Schreibens des Herrn Dr. Eisenstein in Berlin an den Herrn Prof. Richelot in Königsberg, und eines Antwortscbreibens des Herrn Prof. Richelot an den Herrn Dr. Eisenstcin. . . . . . . . . . .

III. 261

17. Einige Bemerkungen zum Eulerschen Additionstheorem der elliptischen Integrale. Von Herrn Dr. Richelot, Prof. ord. an der Univ. zu Königsberg in Pr.

IV. 277

19. Zwei Zahlen-Aufgaben; die erste mit der Auflösung, die zweite noch aufzulösen. Vom Herausgeber. . . . . . . . . . . . . . . . .

21. Recherches sur les coefficients des facultés analytiques. Par Mr. le Dr. O. Schlömilch, Prof. d'Analyse à l'école royale polytechnique de Dresda. IV. 317

24. Aufgabe. .

IV. 344

IV. 376

\section{G e o m e t $\mathrm{r}$ i e.}

1. Erzeugung der Curven vierter Ordnung durch Bewegung gerader Linien. Von Herrn H. Grafsmann, Oberlehrer an der Friedrich-Wilhelms - Schule zu Stettin.

9. Die Brennlinie für den elliptischen Quadranten, wenn die einfallenden Strablen vom Mittelpunct der Ellipse ausgehen und jeder zurückgeworfene Strahl denselben Winkel mit der zugehörigen Normale bildet, wie der entsprechendẹ einfallende. Von Herrn Prof. Dr. Lehmus zu Berlin. . . . . . . .

13. Über die Sätze vom Parallelogramm der Kräfte und vom Hebel; so wie vom Parallelepipedum der Kräfte. Vom Herausgeber. 
15. Kurze Ableitang des Legendreschen Satzes über die Reduction der BeHeft. Seite. rechnung eines sphärischen auf die eines ebenen Dreiecks. Von Herrn A. Winkler, Ingenieur-Practicanten zu Carlsruhe. . . . . . . . .

16. Lehrsătze. Von Herrn Professor J. Steiner zu Berlin. . . . . . . . III. 275

18. Utber Kreiscoordinaten. Von Herrn Dr. W. Stammer aus Luxemburg, Candidaten des hōhern Schulamts. . . . . . . . . . . . . . .

20. Über symmetrische Figuren. Von dem Herrn Prof. Möbiıs zu Leipzig. (Aus den Berichten der Königl. Sächsischen Gesellschaft der Wissenschaften zu Leipzig von 1851.).

IV. 295

23. Geometrisches. Von Herrn $\boldsymbol{W}$. Winkhtats, cand. math. zu Halver bei Arnsberg. IV. 375

\section{3. $\mathrm{M}$ e c ha $\mathrm{n} \mathrm{i} \mathrm{k}$.}

4. Eine neue Lòsung des Problems der Rotation eines festen Körpers um einen Punct. Von Herrn Dr. F. J. Richelot, ordentl. Prof, der Math. an der Universität zu Kōnigsberg. (Auszug einer in der Ferd. Dümmler'schen Buchhandlung zu Berlin 1851 erschienenen Schrift.) . . . . . . . . . .

12. Exposé de diverses remarques et reflexions sur les moments et d'autres sujels de statique. Par Mr. Steichen, professeur à l'école militaire de Bruxelles.

13. Ober die Sãtze vom Parallelogramm der Kräfte und vom Hebel; so wie vom Parạllelepipedum der Krăfțe. Vom Herausgeber. . . . . . . . . . III. 220

\section{Angewandte Mathematik.}

5. Uber einige Grundformeln der Geodãsie. Von Herrn Ferd. Minding, Prof. der. Màth. an der Universitāt zu Dorpat. (Nach dem „Bulletin phys. mathém.” T. VIII. No. 6.)

6. Uber den Umlauf des Springers auf dem Schachbrette (den sogenannten Rôsselsprung). Von Demselben. (Auś dem „Bulletin de la classe phys. math. de l'Académie des sciences de St. Pétersbourg.") . . . . . .

7. Uber die Wirkung des durch eine Drathspirale gehenden elektrischen Stroms auf eine in der Spirale befindliche weiche Eisenmasse. Von Herrn Dr,

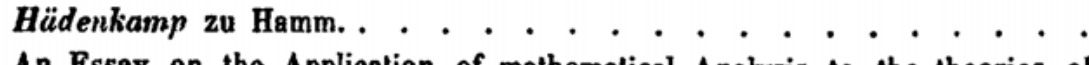

22. An Essay on the Application of mathematical Analysis to the theories of Electricity and Magnetism. By the late George Green, fellow of Gonvilleand Cains-Colleges at Cambridge. (Vide tome 39 p. 13 of this Journal.) 